Bol. Soc. Bot. México 50:19-42 (1990)

\title{
Lista florística de las algas marinas bentónicas de la costa norte de Michoacán, México
}

\author{
Kurt m. Dreckmann, Francisco F. Pedroche y Abel Sentíes G. ${ }^{1}$
}

\begin{abstract}
RESUMEN. Se reportan los resultados de un estudio ficoflorístico realizado durante los años 1980 y 1984 en la porción norte del estado de Michoacán. Se presentan 68 especies distribuídas en 43 géneros pertenecientes a las divisiones Cyanophyta, Chlorophyta, Phaeophyta y Rhodophyta. Se reportan, también, los nuevos registros para el Pacífico mexicano, así como para el Pacífico Tropical mexicano exclusivamente. Asimismo, comentarios sobre habitat, sustrato y estadio reproductivo acompañan a cada taxon reportado.
\end{abstract}

ABSTRACT. The results of a phycofloristic survey made during the years 1980 and 1984, $10 \mathrm{Km}$ South of the northern limit of the state of Michoacan are reported. The results can be summarized in the taxonomic determination of 68 species distributed in 43 genera belonging to divisions Cyanophyta, Chlorophyta, Phaeophyta and Rhodophyta. New records for the Mexican Pacific phycology are reported, as well as those for the Mexican tropical Pacific exclusively. Comments on habitat, sustrate and reproductive state are also given for each taxon.

Es muy probable que la década pasada haya sido testigo de un auge en el interés por el estudio de las algas marinas litorales, y/o bentónicas, sin precedentes tanto en nuestro país como en el mundo entero. Dicho interés se debe a la información creada en los nuevos terrenos de la farmacología marina, biotecnología algal, pesquerías o maricultura algal, agronomía y horticultura. Tales datos hablan del papel cada vez más importante que las algas pueden - y deben- jugar en áreas tan fundamentales para la humanidad como son la salud y la alimentación.

El número de especies algales que se agregan año con año a la lista de recursos marinos valiosos, en uno u otro sentido, sería difícil de calcular dada la gran cantidad de países con litoral que estudian su flora algal y producen información publicada

1 Laboratorio de Ficología, Departamento de Hidrobiología, División de C.B.S., Universidad Autónoma Metropolitana- Iztapalapa. Apdo. Post. 55-535, Méxiço, D.F., 09340, México.

Dreckmann KM, Pedroche FF, Sentíes-G A. 1990. Lista florística de las algas marinas bentónicas de la costa norte de Michoacán, México. Boletín de la Sociedad Botánica de México 50: 19-42. 
en innumerables revistas y memorias alrededor del globo. Con certeza la lista debe ser larga; sin embargo, es necesario establecer un hecho: toda esa inmensa cantidad de información ficológica proviene, en última instancia, de estudios ficoflorísticos locales (uno o dos puntos de la línea costera), regionales (una región biogeográfica definida climáticamente, por ejemplo) o del litoral de todo un país o varios de ellos.Desde luego, varias floras locales dan lugar a una regional y así sucesivamente; es decir: Qué especies viven en un espacio y tiempo dados. O, en otras palabras, dónde se encuentran los recursos algales potenciales del país.

El reducido número de fícólogos marinos que laboran en instituciones de investigación o de educación superior es, en gran medida, la causa de que aún nos encontremos con importantes "vacíos" ficoflorísticos a lo largo de nuestras costas. Las excepciones podrían ser el litoral del Pacífico de la Península de Baja California y el Golfo de California. Estas dos regiones han sido objeto de tan intenso estudio florístico y taxonómico en el pasado, que cuentan con compendios de gran valor, como son los de Dawson $(1944,1966)$, Setchell y Gardner $(1924,1930)$ y Abbott y Hollenberg (1976). Este cúmulo de información, bien sistematizada, ha permitido el reconocimiento y estudio de poblaciones algales a los ficólogos residentes en la Península, en Sonora y Sinaloa. Tales poblaciones corresponden a Macrocystis pyrifera C. Agardh (Laminariales, Phaeophyceae), Gelidium robustum (Gardner) Hollenberg et Abbott (Gelidiales, Rhodophyceae), Gigartina canaliculata Harvey (Gigartinales, Rhodophyceae), Eucheuma uncinatum Setchell et Gardner (Gigartinales, Rhodophyceae) y Porphyra perforata J. Agardh (Bangiales, Rhodophyceae) (Guzmán de Proo et al., 1986). Todas son especies actualmente comercializadas y sometidas a intensos estudios autoecológicos (poblacionales).

El estado del conocimiento ficoflorístico en el Pacífico Tropical mexicano es, por otra parte, totalmente distinto. Pese a que los compendios que tratan sobre la región son tan antiguos como aquellos de J. Agardh (1848) y Harvey (1858) o monumentales como el trabajo monográfico en varios volúmenes de Dawson (1953, 1954, 1960, 1961 a y b, 1962, 1963 a y b) y de Taylor (1945); en su conjunto se refieren a no más de 12 localidades en Oaxaca, Guerrero, Colima, Nayarit y Mazatlán en Sinaloa. Curiosamente, los estudios ficoflorísticos recientes han sido llevados a cabo en las mismas localidades o muy cercanas a ellas. La inmensa mayoría de los estudios ficoflorísticos actuales en la región son Tesis de Licenciatura y/o Maestría; muchos de ellos valiosísimos ya que tratan de puntos intermedios y, de no ser por su muy escasa difusión, constituirían una excelente ayuda para la ubicación de las diferentes poblaciones algales a lo largo de la costa.

La primera vez que el estado de Michoacán fue visitado en 1980, lo fue sólamente con miras a colectar algas y a reconocer su litoral (Dreckmann, 1987). Posteriormente, Flores-Pedroche et al. (1984), realizan nuevas colectas a lo largo del litoral y presentan la primera prospección ficológica del Estado en el Noveno Congreso Mexicano de Botánica. Durante los siguientes 4 años, el grupo de Ficología Marina de la Universidad Metropolitana Unidad Iztapalapa (UAM- I) continuó colectando y aumentando el 
número de localidades a lo largo de los cerca de $289 \mathrm{~km}$ de litoral michoacano (fig 1). Esta labor ha dado como fruto estudios preliminares sobre la Familia Rhodomelaceae (Ceramiales, Rhodophyceae) (Sentíes, 1985) y Clase Chlorophyceae (Correa, 1986) de 3 de esas localidades y un estudio taxonómico de la División Phaeophyta en la localidad de Pichilinguillo (Osorio-Luna, 1989). El presente reporte corresponde a los resultados del primer intento, por parte del grupo, de realización de una flora global (4 Divisiones algales) en una de las localidades: Playa San Telmo, que constituye, per se, una zona en el extremo norte del litoral michoacano.

\section{ÁREA DE ESTUDIO}

Con base en las características fisiográficas del litoral michoacano, Flores-Pedroche $e t$ al. (1984) dividieron la línea costera del Estado en 3 zonas: norte, centro y sur. La zona sur se extiende desde Lázaro Cárdenas hasta Caletilla de Mexcalhuacan (Mapa 1); recibe una fuerte influencia de la desembocadura del Río Balsas y presenta gran cantidad de extensas playas arenosas. La zona centro es la más larga (ca. de $140 \mathrm{Km}$ en línea recta), va desde Mexcalhuacan hasta Playa San Telmo; dominada fundamentalmente por una costa rocosa de tipo "dentado" (en aspecto aéreo), en la cual se presentan gran cantidad de pequeñas bahías (no más de 800-1000 m de boca), que albergan playitas arenosas, separadas unas de otras por varios kilómetros. La zona norte es la más reducida y comprende una longitud de 1400-1500 m alrededor de Playa San Telmo; se caracteriza por un cambio abrupto de playa rocosa a playa arenosa, ésta se extiende hasta la porción norte del Estado de Colima sin grandes manchas de roca. Constituye el último ambiente disponible para el establecimiento algal en el litoral michoacano y fué elegido, por estas circunstancias, como área objeto de estudio del presente reporte. La ubicación de localidades y área de estudio se presentan en la fig. 1.

\section{MATERIAL Y MÉTODO}

El material algal fué colectado durante la época de secas (febrero-abril), separándolos del sustrato utilizando espátulas de albañil y, en caso necesario, cincel y martillo. Las muestras fueron puestas en bolsas de plástico y fijadas inmediatamente en formol al $4 \%$ (diluído en agua marina). Una vez en el laboratorio, fueron transladadas a líquido conservador (formol al 4\%, glicerinado al 5\% y neutralizado con borato de sodio). El análisis taxonómico fué hecho con ayuda de microscópios compuestos y de disección. Los cortes, cuando fueron necesarios, se hicieron con navaja de rasurar. Todas las preparaciones fueron montadas en miel Karo natural con una pequeña cantidad de cristal violeta para aumentar el contraste. Las láminas fueron hechas utilizando la cámara clara y con tinta china, todas ellas realizadas por el primer autor de este trabajo. 


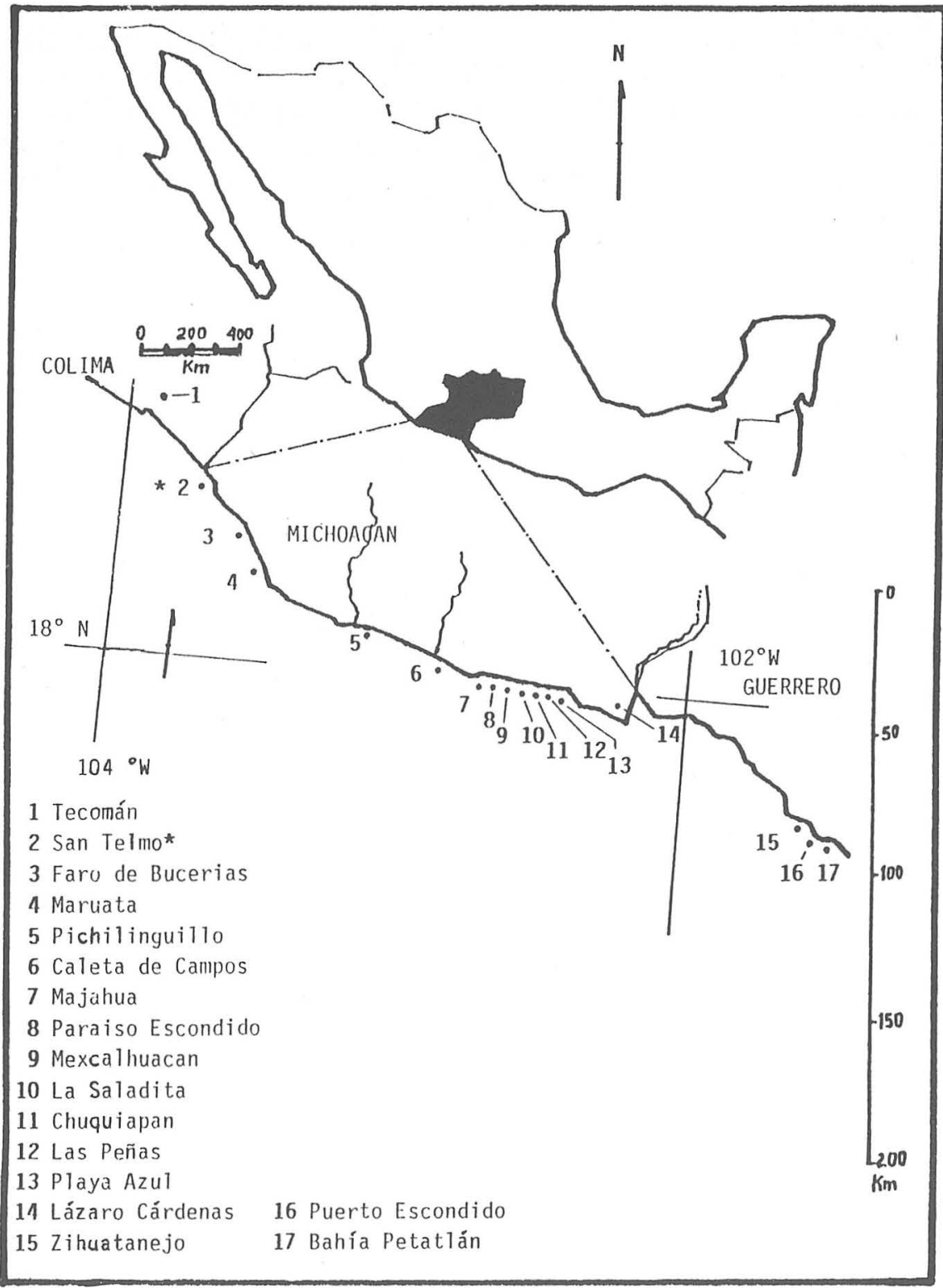

Fig. 1. Ubicación del área de estudio $\left({ }^{*}\right)$ y otras localidades. 
El listado sistemático fué elaborado siguiendo los sistemas de clasificación algal más comunes: Wynne y Kraft (1981), Wynne (1986), Silva et. al (1987) para Chlorophyta, Phaeophyta y Rhodophyta y Desikachary (1959) para Cyanophyta.

Las muestras revisadas en la Colección Ficológica del Herbario de la Facultad de Ciencias de la Universidad Nacional Autónoma de México (FCME) se encuentran depositadas en esa colección bajo los números precedidos por las claves PM, Fm, Rh, $\mathrm{Ph}$, etc.; éstas corresponden a colectas realizadas en Febrero de 1980. Los números de muestra precedidos por la clave FM corresponden a aquellos ejemplares colectados en Abril de 1984 y se encuentran almacenados en la colección ficológica de la Universidad Autónoma Metropolitana-Iztapalapa (UAMI).

\section{RESULTADOS}

A continuación se presenta el listado ficoflorístico de las especies algales encontradas en San Telmo. Este se encuentra arreglado sistemáticamente. Cada taxón es acompañado de un comentario acerca de su abundancia (a partir de su frecuencia de aparición en las muestras y observaciones en el campo). Sólamente los nuevos registros y aquellas especies más abundantes han sido ilustradas (láminas 1-7), ésto para dar una idea de la variación morfológica de las mismas.

\section{División Cyanophyta \\ Clase Cyanophyceae \\ Orden Chamaesiphonales}

Familia Dermocarpaceae

Dermocarpa hemisphaerica Setchell y Gardner. Epífita frecuente sobre Chaetomorpha antennina y especies de Polysiphonia. Herbario: Rh82CA.

Orden Nostocales

Familia Oscillatoriaceae

Lyngbya majuscula Harvey ex Gomont. Abundante. Herbario: PM217. Lámina 1, figura 4.

Lyngbya confervoides C. Ag. ex Gomont. Común como epífita de especies de Cladophora. Herbario: PM217. Lámina 1, figura 5.

Microcoleus sp.. La carencia de ramificaciones en los tricomas, presencia de una vaina común evidente, presencia en algunos tricomas de terminaciones atenuadas y células apicales con caliptra y el habitat marino, permiten asignar este talo al género Microcoleus Desmazieres; sin embargo, las medidas celulares no concuerdan con $M$. chtonoplastes Thuret ex Gomont, la única especie marina reportada por Desikachary (op. cit.). Herbario: FM2. Lámina 1, figura 7. 
y 3 .

Phormidium ambiguum Gomont. Abundante. Herbario: Ph27. Lámina 1, figuras 2

Familia Scytonemataceae

Scytonema rivulare Borzi ex Bornet et Flahault

Forma tapetes de varios centímetros de diámetro. Herbario: FM16, FM16(1). Lámina 1, figura 8.

Familia Microchaetaceae

Microchaete aeruginea Batters. Poco abundante. Herbario: FM16, FM16(1). Lámina 1, figura 6.

Familia Rivulariaceae

Calothrix crustacea Thuret. Muy abundante. Herbario: C21. Lámina 1, figura 9.

División Chlorophyta

Clase Chlorophyceae

Orden Ulotrichales

Familia Ulvaceae

Enteromorpha intestinalis (L.) Link. Muy Abundante. Reportada por Correa (1986) para pichilinguillo (punta rocosa) y Mexcalhuacan (canal de corriente). Herbario: PM140, PM146, PM217.

Enteromorpha compressa (L.) Greville. Abundante. Herbario: FM40.

Enteromorpha flexuosa (Roth) J. Ag.. Abundante. Herbario: FM39 y FM40.

Ulva lactuca L..Muy Abundante.Herbario:PM157.

Familia Cladophoraceae

Orden Cladophorales

Cladophora microcladioides Collins. Escasa. Herbario: FM40.

Cladophora sericea (Hudson) Kützing. Abundante. Herbario: Rh84, PM69, FM84. Lámina 2, figura 4.

Chaetomorpha antennina (Bory) Kützing. Muy Abundante. Correa (1986) la reporta para Pichilinguillo (punta rocosa) y Mexcalhuacan (plataforma rocosa expuesta al oleaje). Herbario: Rh81, FM1, FM3, FM4, FM6, FM7, FM8, FM17, FM23, FM27, FM31, FM32, FM34, FM37, FM43, FM44.

Chaetomorpha californica Collins. Poco abundante. Herbario: PM19, PM241.

Familia Bryopsidaceae

\section{Orden Codiales}

Bryopsis pennatula J. Ag. Muy abundante. Correa (1986) la reporta para Pichilinguillo (plataforma rocosa expuesta y canal de corriente) y Mexcalhuacan (plataforma rocosa semiexpuesta). Herbario: PM160, FM37, FM38, FM39, FM40. Lámina 2, figura 3.

Bryopsis hypnoides Lamouroux. Abundante. Herbario: FM37, FM40, FM49. Lámina 2, figura 2.

Familia Codiaceae

Codium giraffa Silva. Abundante. Herbario: PM133, FM9, FM11, FM14. 
ALGAS DE MICHOACAN

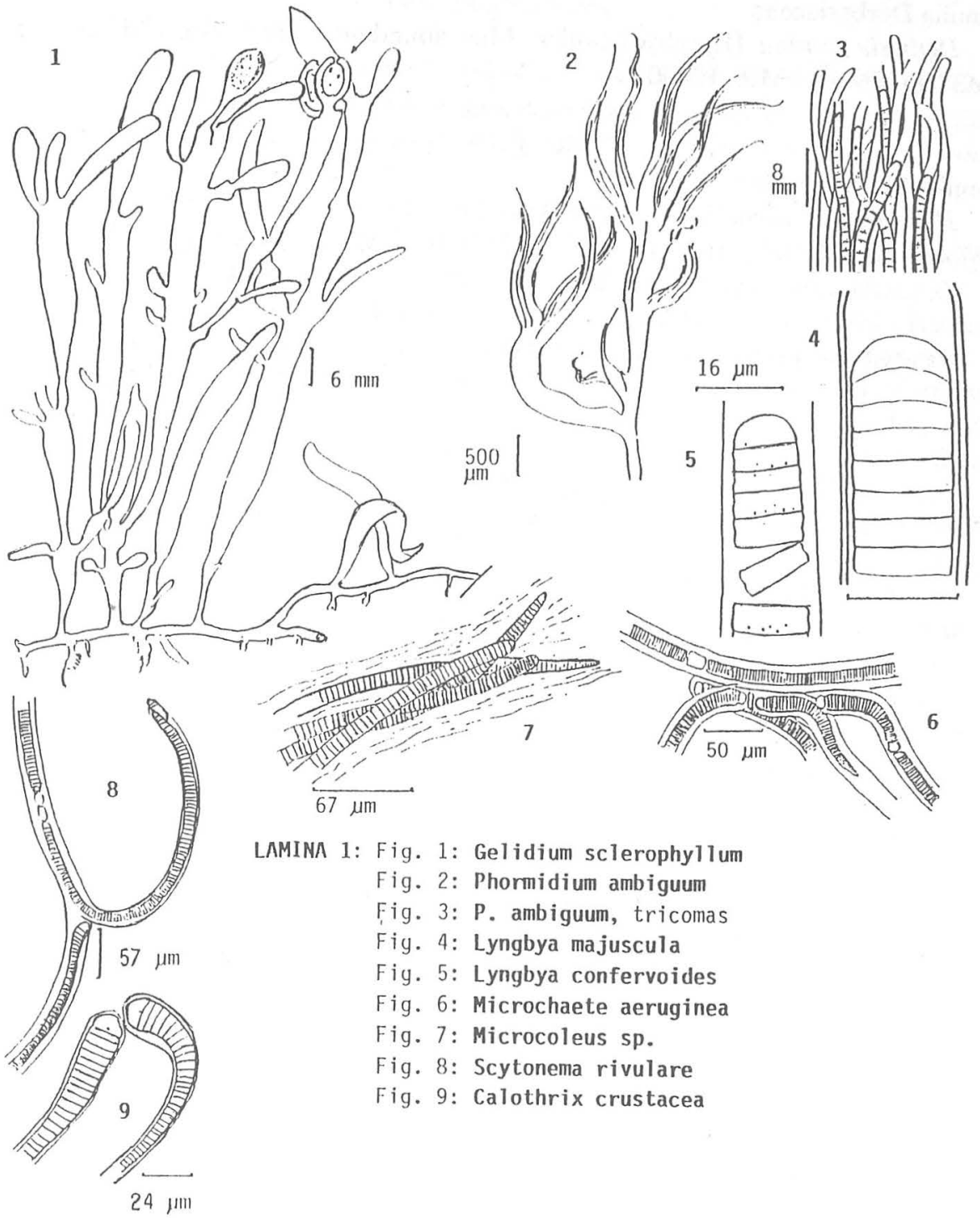


Codium dichotomum (Hudson) S.F. Gray. Escasa. Herbario: FM37, FM46. Lámina 2 , figura 5.

Familia Derbesiaceae

Derbesia marina (Lyngbye) Solier. Muy abundante. Herbario: PM115, FM20, FM37(1), FM38, FM39, FM40.

\section{Familia Ectocarpaceae}

División Phaeophyta

Orden Ectocarpales

Hincksia saundersii (S. y G.) Silva. Abundante. Osorio-Luna (1989) la reporta (como Giffordia saundersii) para Pichilinguillo. Herbario: FM8. Lámina 3, figura 5.

Hincksia sandriana (Zanardini) Silva. Epífita frecuente de Dictyota bartairesii. Herbario: FM3, FM6, FM13, FM18. Lámina 3, figura 7.

Feldmannia indica (Sonder) Womersley y Bailey. Abundante sobre especies de Amphiroa. Osorio-Luna (1989) la reporta para Pichilinguillo. Herbario: FM37, FM38, FM39, FM40.

Familia Ralfsiaceae

Ralfsia hesperia Setchell y Gardner. Muy abundante junto a Chnoospora minima y especies de Chaetomotpha, Hincksia y Grateloupia. Herbario: FM16, FM29, FM42. Lámina 3, figura 6.

Ralfsia pacifica Hollenberg. Frecuente junto a especies de Chaetomorpha, Hypnea, Ceramium y Grateloupia. Osorio-Luna (1989) la reporta para Pichilinguillo. Herbario: FM30.

Ralfsia confusa Hollenberg. Muy abundante, compite por sustrato con otras algas, entre ellas la costrosa (roja) Peyssonnelia mexicana. Osorio-Luna (1989) la reporta para Pichilinguillo. Herbario: FM13, FM15, FM17, FM18.

Petrodema sp.. Las únicas especies descritas por la literatura son $P$. maculiforme Kuckuck (Wynne, 1969; Hollenberg, 1969) y P. steinitzii Rays y Dor (Wynne, 1969). El talo recolectado en el norte de Michoacán no concuerda con ninguna de ellas; por tanto, es muy posible que nos enfrentemos a una nueva entidad (Dreckmann, 1987:146-147). La descripción de los talos recolectados es la siguiente: planta costrosa de color café sepia pálido, fuertemente adherida al sustrato (roca); en corte, se aprecia un grosor de 350-400 $\mu \mathrm{m}$; compuesta de filamentos erectos, separados unos de otros, que surgen de un hipotalo monostromático compuesto de células que miden $10-11 \mu \mathrm{m}$ de diámetro y 25-30 $\mu \mathrm{m}$ de largo; los filamentos erectos (epitalo) mantienen un diámetro de 6.7-7.0 $\mu$ $\mathrm{m}$ a todo lo largo, cada uno de éstos consta de de 10-17(25) células cuadráticas; unangia terminales, de 10.0-11.2 $\mu$ m de diámetro y 25.0-25.5 $\mu$ m de largo. Herbario: FM22, FM31.

Familia Chnoosporaceae

Orden Scytosiphonales

Chnoospora minima (Hering) Papenfuss. Muy abundante. Osorio- Luna (1989) la reporta para Pichilinguillo. Herbario: Ph29, PM231, Ph26, Rh82C, PM191, FM1, FM4, 
FM6, FM7, FM8, FM12, FM13, FM16, FM25, FM37, FM39, FM40, FM46. Lámina 2, figura 1.

Familia Sphacelariaceae

\section{Orden Sphacelariales}

Sphacelaria didichotoma Saunders. Abundante en la flora epífita junto a especies de Hincksia, Centroceras, Bryopsis y Derbesia; en sustratos rocosos ocupados por especies de Chaetomorpha, Chnoospora, Amphiroa, Padina, Grateloupia y Rhodymenia. Herbario: FM31, FM38. Lámina 3, figura 5.

Familia Dictyotaceae

Orden Dictyotales

Dictyota bartayresii Lamouroux. Muy abundante. Herbario: FM2, FM6, FM9, FM11, FM13, FM14, FM18, M19. Lámina 3, figura 4.

Padina durvillaei Bory de Saint-Vincent. Poco abundante. Osorio-Luna (1989) la reporta para Pichilinguillo. Herbario: PM206, PM191, FM38. Lámina 3, figura 2.

Padina vickersiae Hoyt. Muy abundante. Osorio-Luna (1989) la reporta para Pichilinguillo. Herbario: FM3, FM8, FM14, FM19, FM39. Lámina 3, figura 1.

Familia Erythropeltidaceae

División Rhodophyta

Clase Rhodophyceae

Sub Clase Bangiophycidae

Orden Bangiales

Erythrotrichia carnea (Dillwyn) J. Ag.. Epífita abundante de las porciones basales de Feldmannia indica. Herbario: FM40. Lámina 4, figura 1.

Erythrocladia subintegra Rosenvinge. Epífita frecuente, sobre

todo de Chaetomorpha antennina. Herbario: $\mathrm{Ph} 224$.

Orden Goniotrichales

Familia Goniotrichaceae

Goniotrichum alsidii (Zanardini) Howe. Muy abundante sobre Bryopsis hypnoides. Herbario: FM37, FM38.Lámina 4, figura 2.

\section{Sub Clase Florideophycidae \\ Orden Nemalionales}

Familia Acrochaetiaceae

Acrochaetium sinicolum (Dawson) Papenfuss. Escasa. Sobre Ralfsia hesperia. Herbario: FM16, FM29, FM42. Lámina 4, figura 3.

\section{Orden Gelidiales}

Familia Gelidiaceae

Gelidium pusillum (Stackhouse) Le Jolis. Muy abundante, forma tapetes extensos. Herbario:Rh73C,FM37,FM38.Lámina4, figura 5.

Gelidium sclerophyllum Taylor. Poco abundante. Herbario: Rh83, Rh84, FM38. Lámina 1, figura 1. 


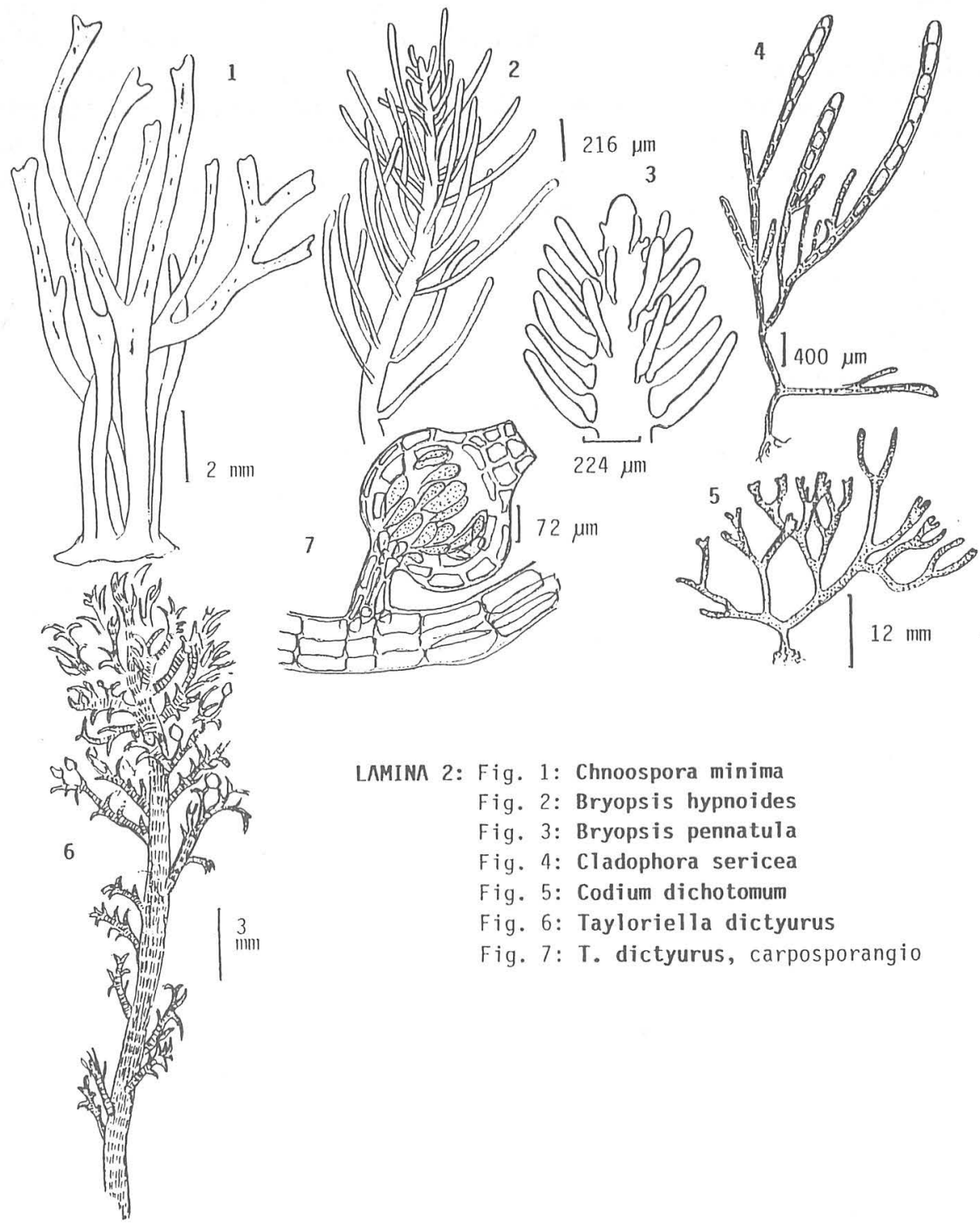



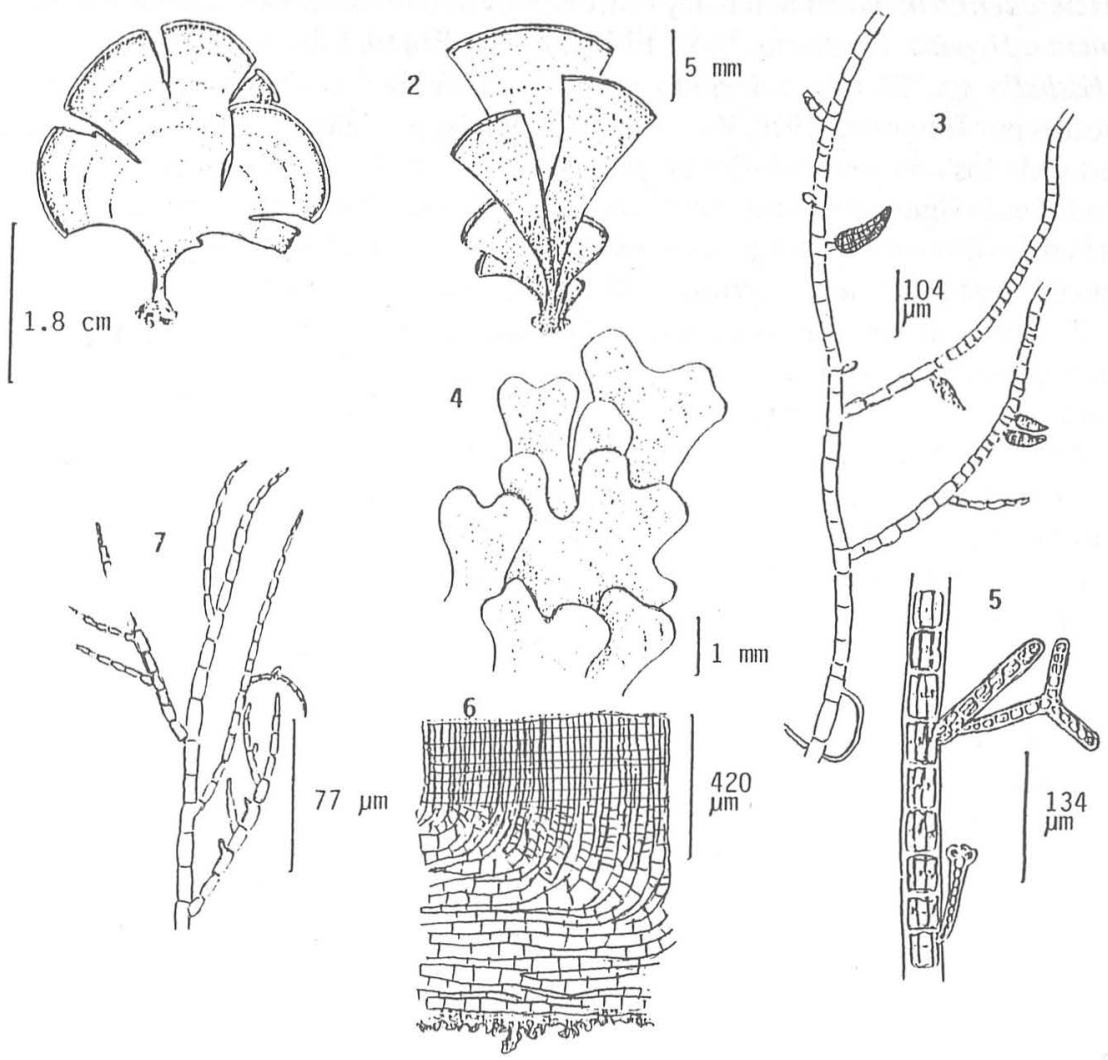

ìMinর 3: Fig. 1: Padina vickersiae, Fig. 2: Padina durvillaei, Fig. 3: Hincksia saundersii, Fig. 4: Dictyota bartayresii, Fig. 5: Sphacelaria didichotoma, Fig. 6: Ralfsia hesperia, Fig. 7: Hincksia sandriana 


\section{Familia Corallinaceae}

\section{Orden Corallinales}

Heteroderma nicholsii Setchell y Mason. Muy abundante sobre especies de Padina, Laurencia e Hypnea. Herbario: FM6, FM14, FM18, FM19. Lámina 4, figura 7.

Fosliella sp.. El talo colectado reune la combinación de caracteres genéricos propuestos por Johansen $(1976,1981)$ : costra delgada, peritalo a veces presente (excepto alrededor de los conceptáculos) y presencia de tricocistos. La descripción de los talos colectados es la siguiente: planta costrosa no ramificada, de color rosado, adherida a las partes basales de especies del género Padina; talo no imbricado y de superficie granular, delgado (menos de $200 \mu \mathrm{m}$ de grosor), de $1-2 \mathrm{~cm}$ en diámetro; costras no transparentes, de 10-15 células de grueso; conceptáculos asexuales de 100-150 $\mu \mathrm{m}$ de diámetro. Muy abundante. Herbario: PM206, FM38. Lámina 4, figura 6.

Jania tenella (Kützing) Grunow. Muy abundante. Herbario: FM1- FM14, FM16FM19, FM33, FM34-FM41.

Amphiroa dimorpha Lemoine. Abundante. Herbario: PM231, Rh83A, Rh78, FM6, FM8, FM18, FM37, FM40.

Amphiroa mexicana Taylor. Abundante. Herbario: PM212, Rh82CB, FM18, FM37. Lámina 5, figuras 5 y 6.

Amphiroa franciscana Taylor. Abundante. Herbario: Rh79B, FM19, FM20.

Orden Gigartinales

Familia Halymeniaceae

Grateloupia doryphora (Montagne) Howe. Muy abundante. Herbario: Rh82E, Rh83, Ph29, FM10, FM46. Lámina 6, figura 1.

Grateloupia abreviata Kylin. Poco abundante. Herbario: Rh77, FM12, FM10, FM14, FM17. Lámina 5, figura 2. FM17.

Grateloupia hancockii Dawson. Abundante. Herbario: Rh77, FM12, FM10, FM14,

Prionitis filiformis Kylin. Muy abundante. Herbario: Rh82B, FM15, FM17. Lámina 4, figura 4.

Familia Peyssonneliaceae

Peyssonnelia mexicana Dawson. Muy abundante. Su calcificación es, principalmente, interna. Herbario: FM2, FM8, FM13, FM17, FM23, FM34.

Familia Kallymeniaceac

Callophyllis violacea J. Ag..Escasa.Herbario: Rh77.

Familia Hypneaceac

Hypnea spynella (C. Ag.) Kür\%ing. Muy abundante. Herbario: FM1-FM11, FM14, FM17, FM 18, FM20, FM27, FM30, FM36, FM40), FM41, FM44.

Jamilia Rhodymeniaceae

()rden Rhodymeniales

Rhodymenia californica Kylin. Abundante. Herbario: Rh83, Rh84, PM216, FM6, IMJ7, IMI8, I M27. 

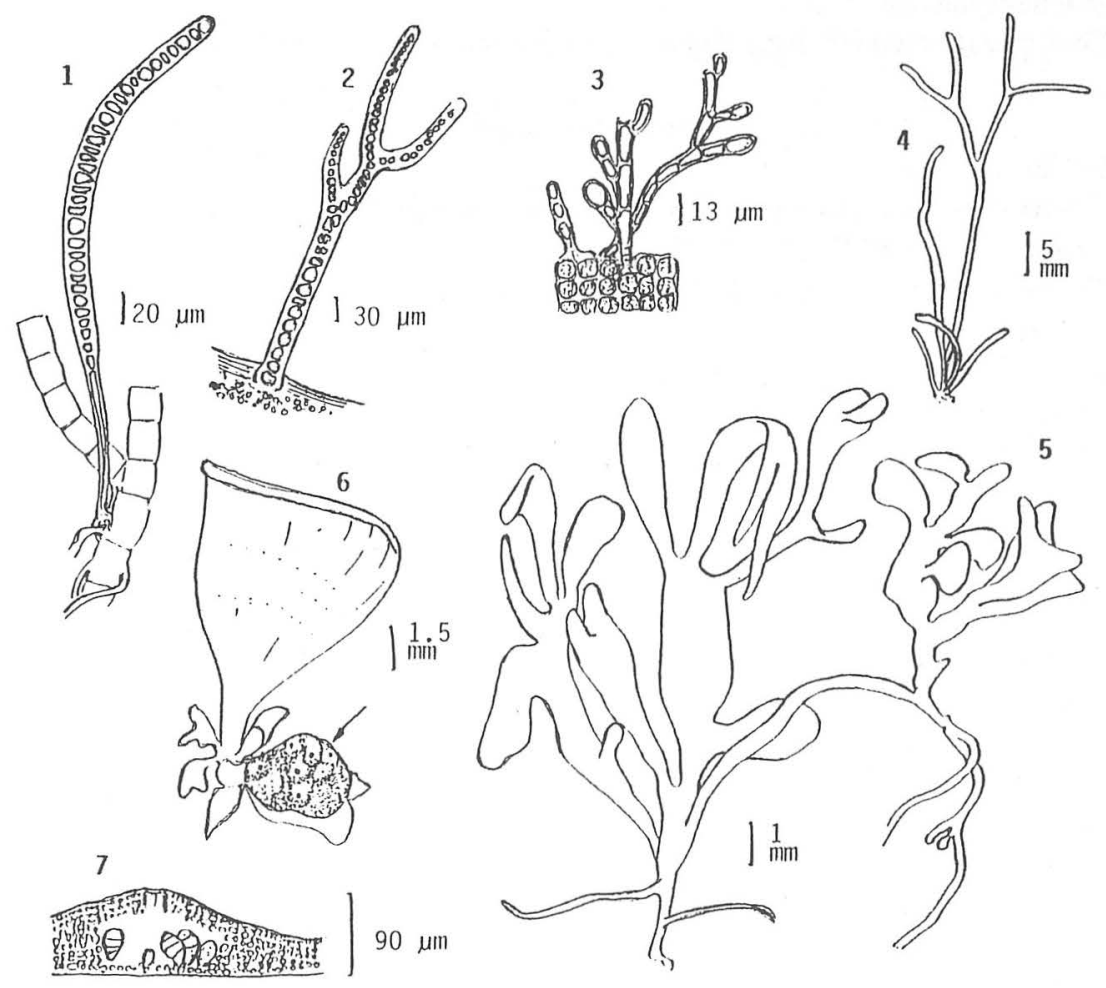

LAMINA 4: Fig. 1: Erythrotrichia carnea, Fig. 2: Goniotrichum alsidii, Fig. 3: Acrochaetium sinicolum, Fig. 4: Prionitis filiformis, Fig. 5: Gelidium pusillum, Fig. 6: Fosliella sp., Fig. 7: Heteroderma nicholsii 
Rhodymenia pacifica Kylin. Abundante. Herbario: Rh80"O", Rh82E, Rh83, FM12, FM34. Lámina 7, figura 2.

Familia Champiaceae 1.

Champia parvula (C. Ag.) Harvey. Abundante. Herbario: FM40. Lámina 5, figura

\section{Familia Ceramiaceae}

\section{Orden Ceramiales}

Centroceras clavulatum (C. Ag.) Montagne. Muy abundante. Herbario: FM1, FM4, FM13-FM17, FM25-FM28, FM36, FM41.

Ceramium comiculatum Montagne. Abundante. Herbario: PM202, FM13, FM14. Lámina 5, figura 4.

Ceramium luetzelburgii Schmidt. Abundante. Herbario: Rh85, PM224, FM16, FM20. Lámina 7, figura 1.

Ceramilum flaccidum (Kützing) Ardissone. Abundante. Herbario: PM224, FM30, FM35, FM39. Lámina 7, figura 3.

Pleonosporium mexicanum Dawson. Muy abundante. Herbario: PM230, Rh85, FM39, FM40, FM46. Lámina 6, figura 2.

Familia Rhodomelaceae

Polysiphonia confusa Hollenberg. Escasa. Sentíes (1985) la reporta para Pichilinguillo (plataforma rocosa). Herbario: FM17.

Polysiphonia scopulonum Harvey. Escasa. Sentíes (1985) la reporta para Mexcalhuacan (canal de corriente). Herbario: Rh85.

Polysiphonia sphaerocarpa Boergesen. Abundante. Sentíes (1985) la reporta para Pichilinguillo (punta rocosa, plataforma rocosa y canal de corrientes). Herbario: FM2, FM3, FM6, FM9, FM18, FM19.

Tayloriella dictyurus (J. Ag.) Kylin. Muy abundante. Sentíes (1985) la reporta para Pichilinguillo (punta rocosa y canal de corriente) y Mexcalhuacan (punta rocosa). Herbario: FM2, FM24, FM26, FM46. Lámina 2, figuras 6 y 7.

Herposiphonia tenella (C. Ag.) Ambronn. Abundante. Sentíes (1985) la reporta para Mexcalhuacan (plataforma rocosa). Herbario: FM40.

Herposiphonia littoralis Hollenberg. Abundante. Sentíes (1985) la reporta para Pichilinguillo (plataforma rocosa). Herbario: Rh73B, FM17.

Chondria arcuata Hollenberg. Abundante. Herbario: PM224, Rh83A, FM7. Lámina 5, figura 3.

Laurencia hancockii Dawson. Abundante. Sentíes (1985) la reporta para Pichilinguillo (plataforma rocosa y canal de corrientes) y Mexcalhuacan (plataforma rocosa). Herbario: FM7, FM16, FM17, FM20, FM21.Lámina 6, figuras 3 y 4.

Laurencia voragina Taylor. Abundante. Sentíes (1985) la reporta para Pichilinguillo (punta rocosa) y Mexcalhuacan (canal de corrientes). Herbario: FM18, FM19, FM20.

De la confrontación de la lista anterior con el Catálogo de Macroalgas del Pacífico mexicano (Pedroche y Silva, en preparación), los siguientes constituyen nuevos registros 

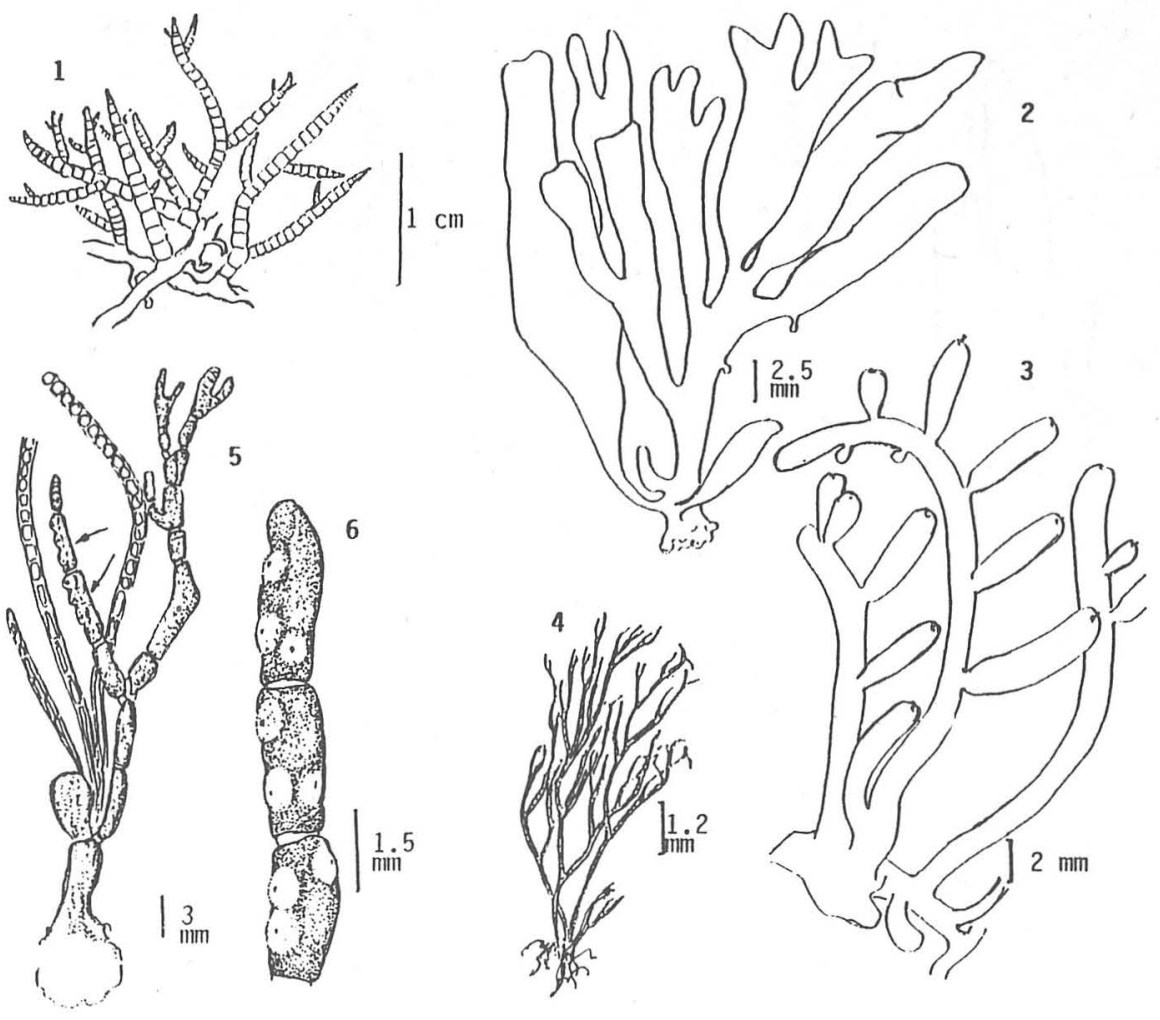

LAMINA 5: Fig. 1: Champia parvula, Fig. 2: Grateloupia abreviata, Fig. 3: Chondria arcuata, Fig. 4: Ceramium corniculatum, Fig. 5: Amphiroa mexicana, Fig. 6: A. mexicana, conceptá culos tetrasporangiales 


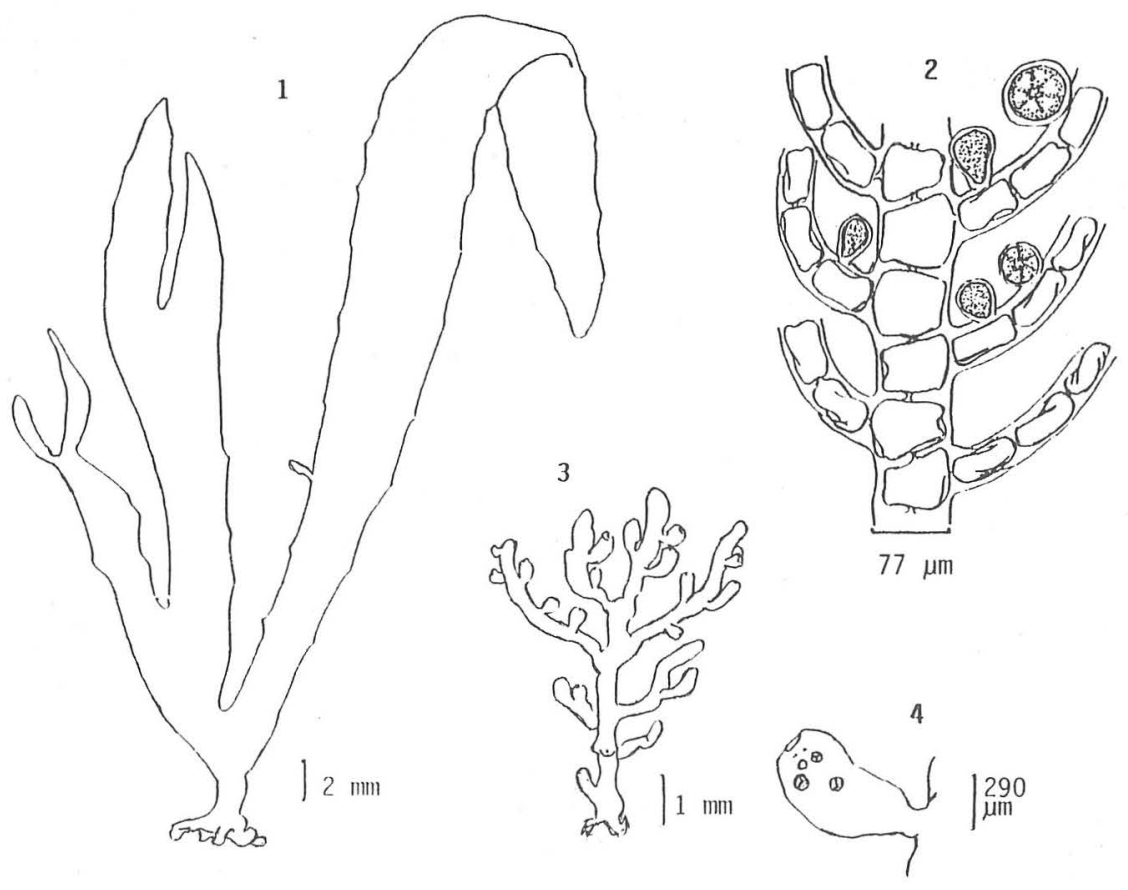

LAMINA 6: Fig. 1: Grateloupia doryphora, Fig. 2: Pleonosporium mexicanum, Fig. 3: Laurencia hancockii, Fig. 4:

L. hancockii, ramita tetrasporangial 

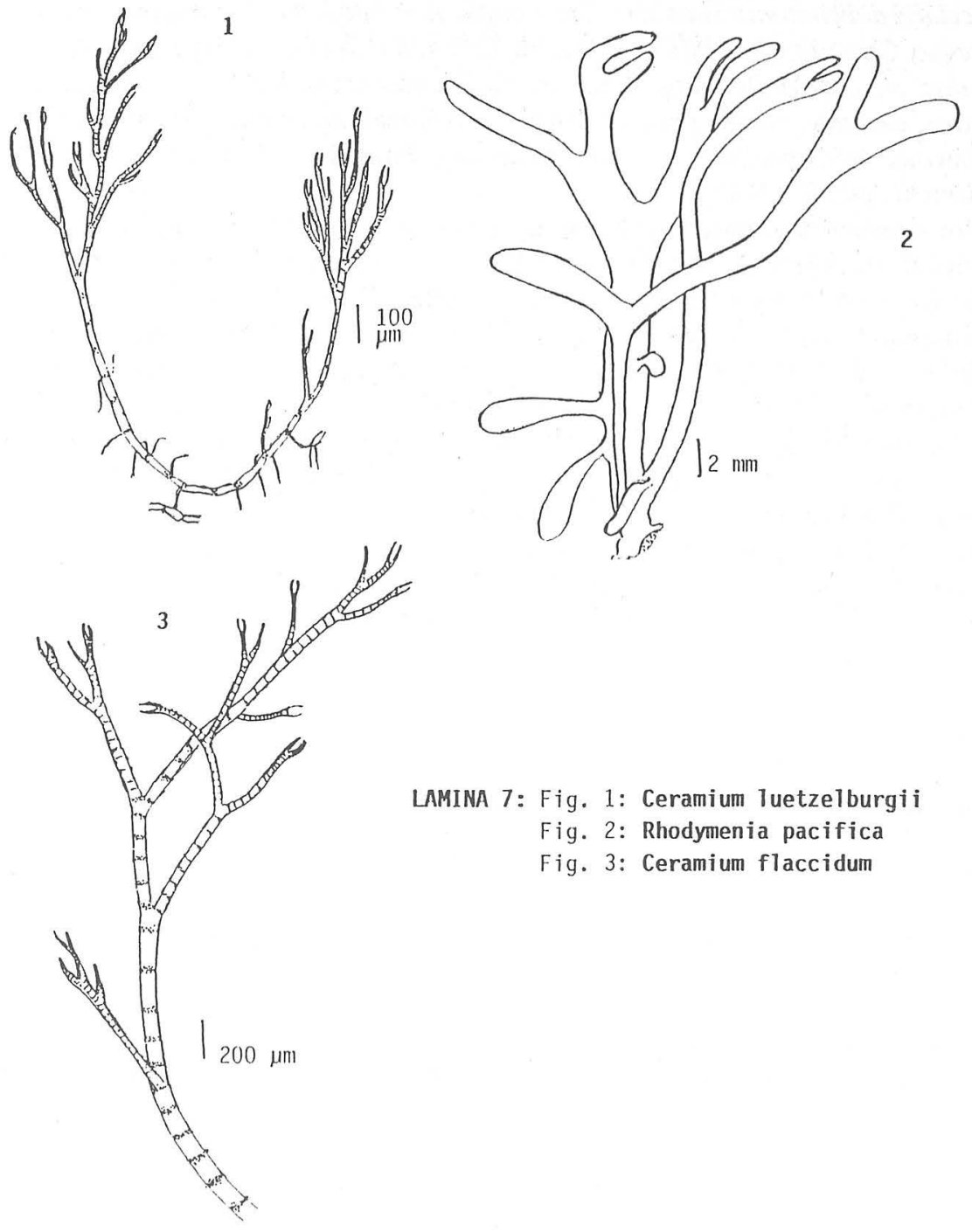

LAMINA 7: Fig. 1: Ceramium Tuetzelburgi i

Fig. 2: Rhodymenia pacifica

Fig. 3: Ceramium flaccidum 
para el Pacífico mexicano:Cladophora sericea (Hud.) Kuetzing, Hincksia saundersii (S. y G.) Silva, Hincksia sandriana (Zan.) Silva, Ralfsia hesperia Setchell y Gardner, Sphacelaria didichotoma Saunders, Grateloupia abreviata Kylin, Ceramium comiculatum Montagne, Ceramium luetzelburgii Schmidt, Ceramium flaccidum (Kuetzing) Ardissone, Chondria arcuata Hollenberg. Además, incluyendo éstas, los siguientes son nuevos registros exclusivamente para el Pacífico Tropical mexicano: Bryopsis hypnoides Lamouroux, Acrochaetium sinicolum (Dawson) Papenfuss, Prionitis filiformis Kylin, Rhodymenia pacifica Kylin.

Por consiguiente, para la porción norte del estado de Michoacán, se reportan 68 especies algales bentónicas; de las cuales 11 (el 7.5\%) constituyen nuevos registros para el Pacífico mexicano y 4 (el 2.7 \%) para el Pacífico Tropical mexicano estrictamente. Considerando que la temperatura media anual superficial del agua en las costas del estado es de $27.9^{0}$ C (SM., Dir. Gral. de Oceanografía, 1978), la ficoflora aquí listada queda comprendida dentro del intervalo de distribución tropical de algas marinas en relación con la temperatura del agua (Setchell, 1915, 1917, 1920). La distribución de las 68 especies en ambiente, sustrato y estado reproductivo se presenta en la Tabla 1. La mayor cantidad de especies es captada por los ambientes risco y poza de marea (Gráfica 1); del mismo modo, los sustratos con mayor número de especies (Gráfica 2) son el rocoso y otras algas (la flora epífita es abundante). Cabe señalar que la mayoría de las especies se encontraron en estado vegetativo (Gráfica 3).

\section{DISCUSIÓN}

La ausencia de especies del género Sargassum en el área de estudio, y por lo menos hasta Pichilinguillo (Osorio Luna, 1989), en contraposición a su abundancia en el litoral de Guerrero y Oaxaca, hacia el sur, y de Jalisco hacia el norte, es un hecho que, agregado al alto número de nuevos registros reportados para esta pequeña área nos hace pensar que la zona alrededor de San Telmo constituye un área de transición fitogeográfica en el gradiente ficoflorístico latitudinal entre las regiones tropical y templada del Pacífico.

Esto se hace más evidente si comparamos listados ficoflorísticos de zonas adyecentes y obtenemos porcentajes de similitud con respecto a San Telmo. Así; hacia el sur, el listado más próximo es el realizado por Chávez (1972) en Zihuatanejo, Gro.: de 65 especies listadas por el autor, el 21.53\% (14) es común a San Telmo. Aproximadamente $20 \mathrm{~km}$ al sur de Zihuatanejo, se encuentra Puerto Escondido, Gro., ahí, Candelaria (1985) reporta 21 especies, de éstas el 52.38\% (11) es común a nuestra área de estudio. De la porción centro-sur de Oaxaca, León Tejera (1986) reporta 49 especies; de éstas el 26.53\% (13) es común a San Telmo. En el Golfo de Tehuantepec, Huerta y Tirado (1970) reportan 85 especies, de las cuales el 17.64\% (15) es común al área de estudio. Hacia el norte, en Chamela, Jal., Flores-Pedroche y González-González (1981) reportan 46 especies, de éstas el 32.60\% (15) es común a San Telmo. Finalmente, hacia el oeste, en 
EDO. REP。

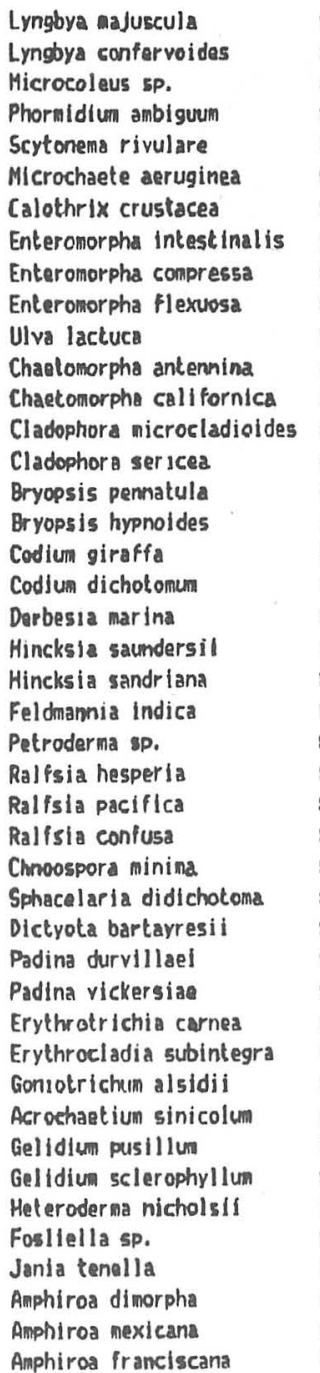

Rs Ac Pr Pm Rx Oq Ps In Es sx Rc. RA Ca Cn E 9 C H Es

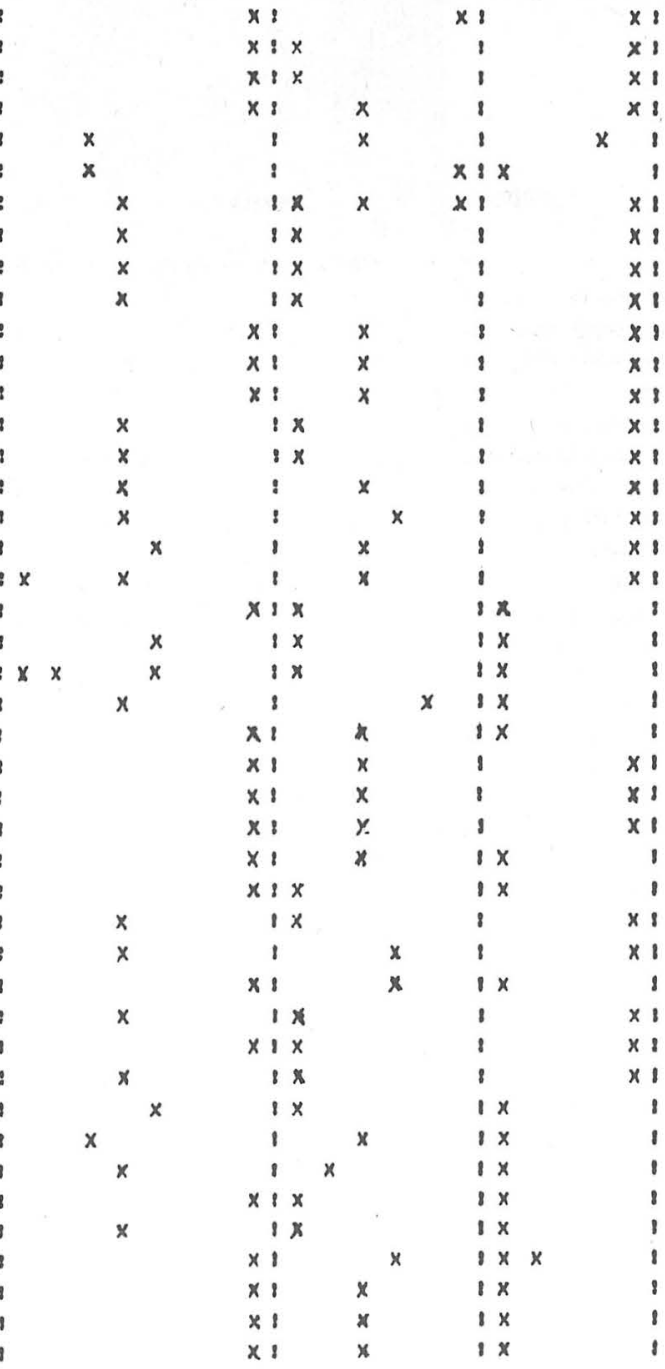


TABLA 1. Continuación.

ESPECIES

AMBIENIES

SUSTRATO

EDO. REP.

\begin{tabular}{|c|c|c|c|c|c|c|c|c|c|c|c|c|}
\hline Grateloupla doryphora & $: x$ & & & & & 8 & & $x$ & & $8 x$ & $x$ & 8 \\
\hline Grateloupla abreviata & 8 & & & $x$ & $x$ & 8 & & $x$ & & 1 & & $\times 1$ \\
\hline Grateloupia hancockii & 8 & & & & & $\times 1$ & & $x$ & $x$ & 1 & & $x$ \\
\hline Prionitis filiformis & $\& x$ & & & & & 1 & & $x$ & & 8 & $x$ & 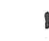 \\
\hline Peyssonnelia mexicana & 8 & & & & & $\times 1$ & & $x$ & & $\times 8$ & & $x$ \\
\hline Callophyllis violacea & $8 x$ & & & & & 8 & & & $x$ & b & $x$ & 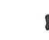 \\
\hline Hypnea spinella & $:$ & & & & & $x:$ & & $x$ & & 1 & & $x$ \\
\hline Rhodymenia californica & $: x$ & & & $x$ & & $\ell$ & & $x$ & & 8 & $x$ & \\
\hline Rhodymenla pacifica & $: x$ & & $x$ & & & 1 & & & $x$ & 1 & $x$ & \\
\hline Champla parvula & : & & $x$ & & & & $x$ & & $x$ & 1 & & $x$ \\
\hline Centrocepss clavulatun & 8 & & & & & $\times 1$ & $x$ & $x$ & & $1 \times$ & $x$ & \\
\hline Ceramium corniculatum & 1 & $x$ & & $x$ & & & $x$ & & & $1 x$ & & 1 \\
\hline Ceramium luetzelburgil & 1 & & & & & 1 & $x$ & & & $1 x$ & & 8 \\
\hline Ceranium flacciorm & $g$ & $x$ & $x$ & & & 1 & $x$ & & & $1 x$ & & 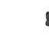 \\
\hline Pleonospor 1 th mexicanum & 1 & $x$ & & & & 1 & $x$ & $x$ & & $1 x$ & & 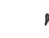 \\
\hline Polvsiphonia confusa & $:$ & & & & $\dot{x}$ & 1 & $x$ & & & $1 x$ & & , \\
\hline Polysiphonia scopulorun & 8 & & & & & 1 & & & $x$ & I & $x$ & 8 \\
\hline Polysiphonia sphaerocarpa & $: x$ & & & & $x$ & 8 & $\times x$ & & & 8 & $x$ & 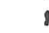 \\
\hline Tayloriells dictyurus & $1 x$ & $x$ & & & & 8 & $x$ & $x$ & & $1 x$ & $x$ & 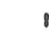 \\
\hline Herposiphonta littoralis & 8 & & & & $x$ & 8 & & $x$ & & 1 & & $\times 1$ \\
\hline Herposiphonis tenella & $\ell$ & & $x$ & & & & $x$ & & $x$ & 1 & $x$ & 8 \\
\hline Chondela arcuata & 8 & & & $x$ & & 8 & & & $x$ & 1 & $x$ & 8 \\
\hline Laurencia hancockil & 8 & & & & $x$ & 8 & & $x$ & & $1 x$ & & 8 \\
\hline Laurencia voragina & 1 & & & & $x$ & $\ell$ & & $x$ & & 8 & & $x:$ \\
\hline
\end{tabular}

CLAVE: Rs=Risco, Ac=Acantilado, Pc=Punta rocosa, Pm= Poza

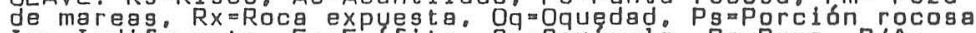
$I n=$ Indiferente. Eg=Eplfita, $S x=5 a x$ Coli, Rc=Roca。 R/A

Roca/Arena. Ca=Carbonato de calcio. Cnm Conchas. E=Esporan giog S=Espermatangio. CmCistocarpo. H=Hormogonio. Eg=Vege 

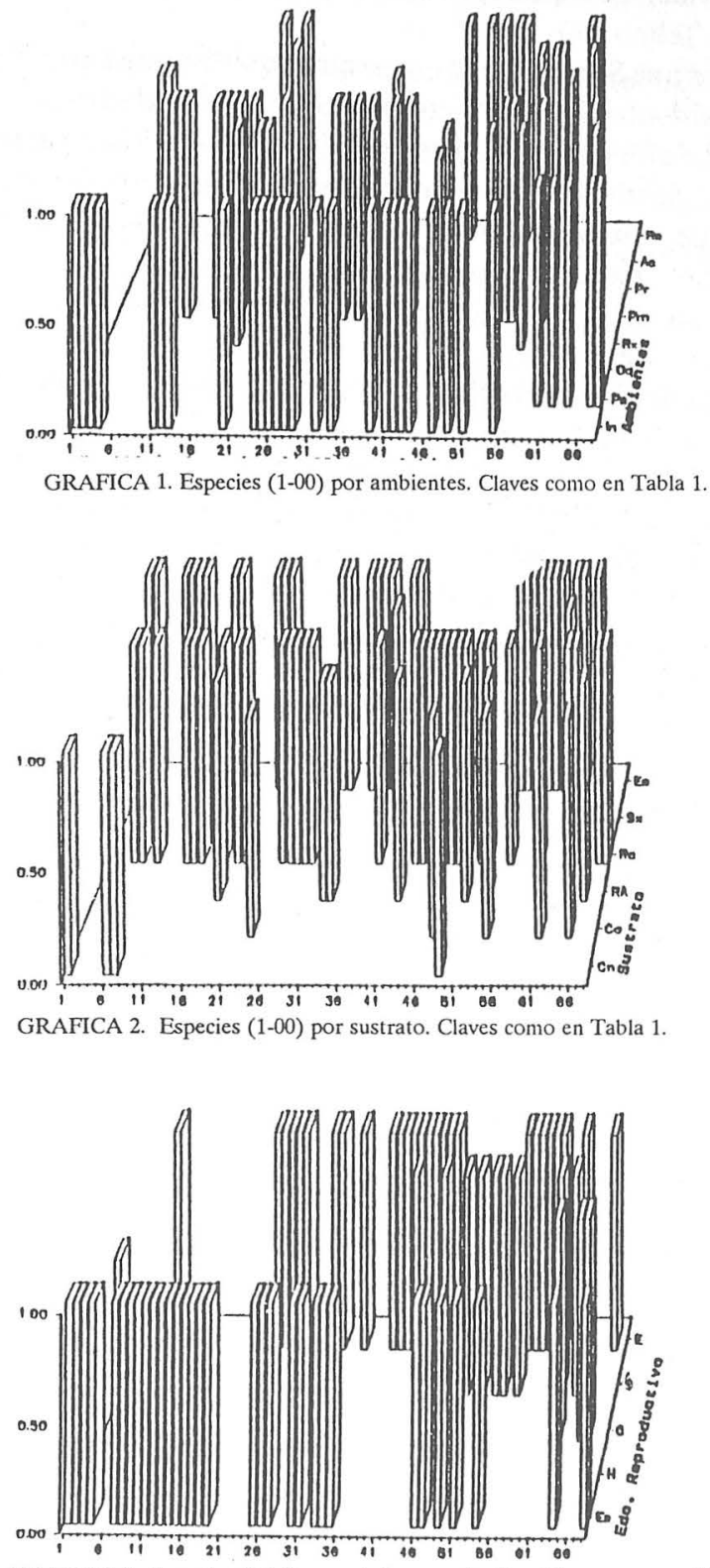

GRAFICA 3. Especies (1-00) por estado reproductivo. Claves como en Tabla 1. 
el Archipiélago Revillagigedo, Col., Huertá y Garza (1975) listan 69 especies, el 10.14\% (7) es común a San Telmo.

Si consideramos que San Telmo se encuentra equidistante entre Zihuatanejo y Bahía Chamela, resulta evidente el incremento paulatino de afinidad en sentido sur-norte y el aumento, también en afinidad, más brusco a partir de San Telmo hacia en norte. La baja afinidad con Revillagigedo se explica no sólo por la gran barrera oceánica que significa la distancia, sino que, también, por las corrientes templadas que bajan de California y cambian su dirección hacia el oeste precisamente frente al área de estudio; estas corrientes significan una barrera accesoria al intercambio ficoflorístico entre el Archipiélago y el area de estudio.

Los porcentajes de similitud existentes entre Puerto Escondido y la zona sur-centro de Oaxaca con San Telmo se aprecian notoriamente superiores al presente entre Zihuatanejo y San Telmo. Esto se debe a que ambos listados son de tipo ambiental, no local; es decir, Candelaria (1985) caracterizó sólamente la ficoflora de los riscos; por su parte, León- Tejera (1986) tomó en consideración exclusivamente las especies algales presentes en las pozas de marea. En este sentido, podemos hacer un ejercicio de similitud porcentual entre ambientes algales si computamos las especies de ambos ambientes en San Telmo y las comparamos con las localidades sureñas. Así; de las 21 especies presentes en los riscos de Puerto Escondido, el $4.76 \%$ es común con las 9 especies que viven exclusivamente en los riscos de San Telmo (ver Gráfica 1). Del mismo modo, de las 49 especies de pozas de marea del centro-sur de Oaxaca, el 6.12\% es común con las 20 especies encontradas en el mismo ambiente en San Telmo. El ejercicio, por si solo, no arroja información valiosa pues no contamos con otros estudios de ficofloras ambientales que nos permita un análisis comparativo. Sin embargo, en la Gráfica 1, se aprecia que los dos ambientes algales que mayor número de especies captan son riscos y pozas de marea. Es decir, estos dos ambientes pueden constituir unidades de estudio ficoflorístico útiles en el análisis del comportamiento de la ficoflora a lo largo del gradiente latitudinal. Por último, de las especies algales que viven en San Telmo, Chetomorpha antennina, Padina durvillaei, Gelidium sclerophyllum, Grateloupia abreviata y Tayloriella dictyumus han mostrado actividad antibacteriana, antifúngica y antimitótica positiva (Sobrino-Figueroa, com. pers.); de donde, el conocimiento ficoflorístico de la localidad en cuestión ha abierto ya las puertas al estudio de las algas como un recurso natural con posibilidades de aprovechamiento.

AGRADECIMIENTOS. Los autores desean expresar su agradecimiento a María Elena Sánchez Rodríguez (E.N.C.B. del I.P.N.) y M. en C. Rosaura Grether (Dpto. de Biología, UAM-I) por sus valiosos comentarios al presente trabajo. 


\section{LITERATURA CITADA}

ABBOTT, I.A. y HOLLENBERG, G. 1976. The Marine algae of California. Stanford University, California. USA. 827p.

AGARDH, J.G. 1847. Nya Alger fran Mexico. Ofvers. af kongl. Vetensk. Akad. Forhandl. 4:5-17

CANDELARIA, C. 1985. Caracterización de la ficollora de la localidad de Puerto Escondido, Guerrero. Tesis, Facultad de Ciencias, UNAM, México, D.F.. 150p.

CHÁVEZ, M.L. 1972. Estudio de la flora marina de la bahía de Zihuatanejo y lugares adyacentes. Mem. IV Congr. Nac. Ocean. (México):265-271.

CORREA, M.Z.E. 1986. La División Chlorophyta en las costas del estado de Michoacán, México. Tesis, Facultad de Ciencias, UNAM, México, D.F. 72p.

DESIKACHARY, T.V. 1959. Cyanophyta. Pub. by Indian Council of Agricultural Research, New Delhi. $686 \mathrm{p}$.

DAWSON, E.Y. 1944. The marine algae of the Gulf of California. Allan Hancock Pacific Expedition 3:189-441.

DAWSON, E.Y. 1953. Marine red algae of Pacific Mexico. Part 1. Bangiales to Corallinaceae, Subf. Corallinoideae. Allan Hancock Pacific Expedition 17:1-239.

DAWSON, E.Y. 1954. Marine red algae of Pacific Mexico. Part 2. Cryptonemiales (cont.). Allan Hancock Pacific Expedition 17:241-397.

DAWSON, E.Y. 1960. Marine red algae of Pacific Mexico. Part 3. Cryptonemiales, Subf. Melobesioideae. Pacific Nature 2:3-125.

DAWSON, E.Y. 1961a. Marine red algae of Pacific Mexico. Part 4. Gygartinales. Pacific Naturalist 2:191-343.

DAWSON, E.Y. 1961b. Marine red algae of Pacific Mexico. Part 5. Polysiphonia. Pacific Naturalist 2:345-375.

DAWSON, E.Y. 1962. Marine red algae of Pacific Mexico. Part 7. Ceramiales: Ceramiaceae, Deleseriaceae. Allan Hancock Pacific Expedition 26:1-207.

DAWSON, E.Y. 1963a. Marine red algae of Pacific Mexico. Part 6. Rhodymeniales. Nova Hedwigia 5:437-476.

DAWSON, E.Y. 1963b. Marine red algae of Pacific Mexico. Part 8. Dasyaceae, Rhodomelaceae. Nova Hedwigia 6:401-481.

DAWSON, E.Y. 1966. New records of marine algae from the Gulf of California. J. Arizona Acad. Sci. 4:55-66.

DRECKAMNN, K.M. 1987. Algas marinas bénticas de Playa San Telmo, Michoacán, México. Tesis Facultad de Ciencias, UNAM, México, D.F.. 170p.

FLORES-PEDROCHE, F. y GONZÁLEZ-GONZÁLEZ, J. 1981. Lista florística preliminar de las algas marinas de la región sur de la costa de Jalisco, México. Phycol. Lat-amer. 1:60-71.

FLORES-PEDROCHE, F., CORREA, Z., DRECKMANN-ESTAY, K. y SENTÍES-GRANADOS, A. 1984. Prospección ficológica de las costas del estado de Michoacán, Méx. Resumenes de IX Congreso Mexicano de Botánica. Sociedad Botánica de México A.C. 278p.

GUZMÁN DEL PROO, S.A., CASAS VALDEZ, M., DÍAZ CARRILLO, A., DÍAZ LÓPEZ, M.L., PINEDA BARRERA, J. y SÁNCHEZ RODRÍGUEZ, M.E. 1986. Diagnóstico sobre las investigaciones y explotación de las algas marinas en México. Investigaciones Marinas CICIMAR 3(Núm. Esp. II):1-63.

HARVEY, W.A. 1858. Nereis Boreali-Americana. Parts I, II y III. Pub. by The Smithsonian Institution, N.Y.

HOLLENBERG, J.G. 1969. An account of the Ralfsiaceae (Phaeophyta) of California. J. Phycol. 5:290-301. 
HUERTA, M.L. y TIRADO, L.J. 1970. Estudio florístico-ecológico de las algas marinas de la costa del Golfo de Tehuantepec, México. Bol. Soc. Bot. México 31:113-137

HUERTA, M.L. y A. M. GARZA BARRIENTOS, 1975. Contribución al conocimiento de la flora marina de las Islas Socorro y San Benedicto del Archipielago Revillagigedo, Colima, Méx.. Bol. Inform. Inst. Bot. Univ. Guadalajara 2(4):4-16.

JOHANSEN, W.H. 1976. Currents status of generic concepts in Coralline algae (Rhodophyta). Phycologia, 15:221-244.

JOHANSEN, W.H. 1981. Coralline algae, A first synthesis. CRC Press, Boca Raton, Florida, 239p.

LEÓN TEJERA, H.P. 1986. Ficoflora de las Pozas de marea de la costa de Oaxaca: Una proposición metodológica. Tesis de Maestría, Facultad de Ciencias, UNAM, México, D.F. 150p.

OSORIO LỤNA, M. 1989. Estudio taxonómico de la División Phaeophyta en la localidad de Pichilinguillo, Michoacán. Reporte de Servicio Social, Depto. Hiđrobiología, División CBS, UAM-Iztapalapa, México, D.F. 60p.

SECRETARÍA DE MARINA, DIR. GRAL. DE OCEANOGRAFÍA. 1978. Temperatura y salinidad de los puertos de México en el Océano Pacífico. Editado por la Dirección General de Oceanografía de la Secretaría de Marina, México, D.F.

SENTÍES, G.A. 1985. Estudio florístico preliminar de la familia Rhodomelaceae (Ceramiales, Rhodophyta) en la costa del estado de Michoacán, Méx. Tesis Facultad de Ciencias, UNAM, México, D.F. 53p.

SETCHELL, A.W. 1915. The Law of temparature connected with the distribution of the marine algae. Ann. Missouri Bot. Gard. 2:287-305.

SETCHELL, A.W. 1917. Geographical distribution of marine algae. Science, 45:197-204.

SETCHELL, A.W. 1920. The temperature interval in the geographical distribution of marine algae.

SILVA, P.C., MENEES, E.G. y MOE, R.L. 1987. Catalog of the benthic marine algae of the Philippines. Smithsonian Contr. Mar. Sci. 27:1-156.

TAYLOR, W.R. 1945. Pacific Marine algae of the Allan Hancock Expedition to the Galapagos Islands. Allan Hancock Pacific Expedition 12:1-528

WYNNE, M.J. 1969. Life history and systematics studies of some Pacific north American Phaeophyceae (Brown Algae). Univ. Calif. Publ. Bot. 50:1-88.

WYNNE, M... 1986. A Checklist of benthic marine algae of the tropical and subtropical western atlantic. Can. \. Bot. 64:2239-2281.

WYNNE, M.J. y KRAFT, G.T. 1981. Classification sumary. En: Lobban, C. S. y M. J. (Edrs.). The biology seaweeds. Blackwell Scientific Publ., London pp.743-760. 